\title{
L'origine virale du SIDA: de la clinique à la biologie moléculaire
}

En moins de quatre ans, épidémiologistes, cliniciens, immunologistes, virologistes et biologistes moléculaires ont démontré l'origine virale du SIDA, identifié le virus, mis au point des tests diagnostiques applicables à la pratique quotidienne et ouvert la voie à l'élaboration d'un vaccin.

\section{David Klatzmann}

Assistant à la faculté de médecine Pitié-Salpétrière.

\section{Luc Montagnier}

Directeur de recherche au CNRS. Institut Pasteur

\section{ABRÉVIATIONS}

SIDA : Syndrome d'immunodéficience acquise.

AIDS : Acquired immunodeficiency syndrome.

LAV : Lymphadenopathy associated virus.

HTLV : Human T-cell leukemia virus.

ARV : AIDS related virus.

EIAV : Equine infectious anemia virus.

MMTV : Mouse mammary tumor virus.

ELISA : Enzyme

linked

immuno-sorbent assay.

RIPA : Radio-immuno precipitation assay.

\section{ADRESSES}

L. Montagnier: Institut Pasteur. 25-28, rue du Docteur-Roux. 75724 Paris Cedex 15.

D. Klatzmann: Faculté de médecine PitiéSalpétrière. 91, bd de l'Hôpital. 75013 Paris. $\mathrm{m} / \mathrm{s}$ mai 85 a survenue d'infections parasitaires, virales ou fongiques, ainsi que le développement d'une tumeur cutanée rare, le sarcome de Kaposi, chez de jeunes homosexuels américains, ont permis de définir une nouvelle entité clinique : le syndrome d'immunodéficience acquise ou SIDA (en anglais AIDS). On sait maintenant que cette maladie n'est pas limitée aux homosexuels, mais atteint également les hémophiles, certains toxicomanes, les Haitiens, les Africains des pays d'Afrique équatoriale et maintenant, de plus en plus, les personnes qui reçoivent des transfusions sanguines.

$\mathrm{Ni}$ les tumeurs ni les infections rencontrées chez ces malades ne sont nouvelles. C'est leur évolution, souvent foudroyante, liée à une profonde et irréversible dépression de l'immunité cellulaire, qui est nouvelle. Ainsi le sarcome de Kaposi était connu depuis longtemps en Afrique et dans les pays du bassin méditerranéen, où il atteint surtout des hommes âgés de plus de 60 ans et a une évolution lente. Il peut se développer aussi de façon transitoire chez les personnes ayant reçu un traitement immunodépresseur à des fins de transplantation d'organe mais régresse à l'arrêt du traitement. Dans le cas du SIDA, le sarcome de Kaposi est au contraire rapidement envahissant. On note aussi chez certains malades atteints du SIDA l'apparition de lymphomes intracérébraux.
Quant aux infections, elles sont dues à des germes dits " opportunistes " car ils se développent et envahissent tissus et cellules lorsque l'immunité cellulaire de l'hôte est défaillante: ainsi les pneumonies à Pneumocystis Carini, les candidoses graves atteignant les muqueuses digestives, les abcès intracérébraux dus aux toxoplasmes, les méningites à cryptocoques, sont parmi les plus fréquentes de ces infections.

\section{Immunologie}

Les tests immunologiques confirment qu'il existe une dépression profonde de l'immunité cellulaire chez de tels malades $[\mathbf{I}, 2]$. Il existe une diminution importante du nombre absolu des lymphocytes dits $\mathrm{T}_{4}{ }^{+}$qui possèdent un marqueur de surface spécifique reconnu par certains anticorps monoclonaux $\left(\mathrm{OKT}_{4}\right.$, Leu 3, etc.). Ces lymphocytes ont un rôle central dans la réponse immunitaire. Ils activent les lymphocytes $T$ effecteurs et les lymphocytes B qui synthétisent des anticorps spécifiques en réponse à un antigène. L'autre grand groupe de lymphocytes $T$, les $T 8^{f}$, dits supresseurs ou cytotoxiques (reconnus par les anticorps monoclonaux de type OKT8 ou Leu 2) est également touché, mais à un moindre degré, de sorte que le rapport $\mathrm{T}_{4} / \mathrm{T} 8$ des lymphocytes circulants, qui normalement est supérieur à I , s'inverse et peut éventuellement devenir très bas. 


\section{REFERENCES}

I. Cavaille-Coll M, Messiah A, Klatzmann D, et al. Critical analysis of $T$-cell subset and function evaluation in patients with persistent generalized lymphadenopathy in groups at risk for AIDS. Clin Exp Immunol 1984; 57: 51 1-9.

2. Gluckman JC, Klatzmann D, Cavaille-Coll $\mathbf{M}$, et al. Is there a correlation of $\mathrm{T}$-cell proliferation functions and surface marker phenotypes in patients with AIDS or lymphadenopathy syndromes? Clin Exp Immunol 1985, in press.

3. Poiesz BJ, Ruscetti FW, Reitz MS, Kayanaraman VS, Gallo RC. Isolation of a new type $C$ retrovirus (HTLV) in primary uncultured cells of a patient with Sézary T-cell leukemia. Nature 1981; 294: 268-71.

4. Barré-Sinoussi F, Chermann JC, Rey F, et al. Isolation of a T-lymphotropic retrovirus from a patient at risk of Acquired Immune Deficiency Syndrome (AIDS). Science 1983; 220: 868-71.

5. Montagnier L, Chermann JC, Barré-Sinoussi $\mathrm{F}$, et al. A new human T-lymphotropic retrovirus: characterization and possible role in lymphadenopathy and acquired immune deficiency syndromes. In: Gallo RC, Essex ME, Gross L, eds. Human $T$ cell leukemiallymphoma viruses. New York: Cold Spring Harbor, 1984: 363-79.

6. Kalyanaram VS, Sarngadharan MG, RobertGuroff $M$, et al. A new subtype of human T-cell leukemia virus (HTLV-II) associated with a T-cell variant of hairy cell leukemia. Science 1983 ; 218: 571-3.

7. Montagnier L, Krust B, Clavel F, et al. Identification and antigenicity of the major envelope glycoprotein of lymphadenopathy associated virus (LAV). Submitted, 1985

8. Alizon $\mathrm{M}$, Sonigo $\mathrm{P}$, Barré-Sinoussi $\mathrm{F}$, et al. Molecular cloning of lymphadenopathy associated virus (LAV). Nature 1984; 31 2: 757-60.

9. Wain-Hobson S, Sonigo P, Danos O, Cole S, Alizon $M$. Nucleotide sequence of the AIDS virus, LAV. Cell 1985; 40: 9-17.
D'autres tests mettent en évidence une altération fonctionnelle de ces lymphocytes. L'injection souscutanée d'antigènes solubles (tuberculine par exemple) n'aboutit plus à la réaction inflammatoire habituellement observée. Elle dépend de la production de médiateurs solubles par les lymphocytes $\mathrm{T}_{4}{ }^{+}$. L'activation et la prolifération lymphocytaires induites par les lectines comme la phytohémaglutinine, sont diminuées ou inexistantes. Il existe aussi une activation polyclonale des lymphocytes B qui se traduit par une hypersécrétion globale d'immuno-globulines, surtout IgG, dont le taux sérique est doublé, voire triplé. En revanche, la synthèse d'anticorps spécifiques contre des antigènes nouveaux est perturbée. Ces altérations peuvent être considérées comme des conséquences de la défaillance des cellules $T$ qui jouent un rôle important dans la régulation de la production des anticorps, bien qu'une action directe de l'agent causal sur les cellules B ne puisse être exclue.

On s'attendait donc à ce que la cible primaire de l'agent étiologique supposé du SIDA soit constituée par les lymphocytes $\mathrm{T}_{4}{ }^{+}$.

\section{A la recherche d'un virus}

Les observations épidémiologiques ont rapidement montré que le SIDA était une maladie transmissible. L'apparition des premiers cas chez des malades hémophiles, contaminés par les transfusions de facteurs anti-hémophiliques; a orienté les recherches vers un virus, seul agent infectieux pouvant passer à travers les filtres utilisés pour la préparation de ces facteurs. Les premières recherches ont concerné des virus connus, essentiellement de la famille des Herpès virus, mais ont été infructueuses. En effet, aucune souche particulière de ces virus pouvant expliquer la nouveauté de la maladie n'a pu être isolée chez les malades. Les recherches se sont alors orientées vers la famille des rétrovirus, d'autant qu'il existait un modèle animal séduisant, celui de la leucémie du chat. En effet, le rétrovirus à l'origine de cette leucémie peut également causer chez les ani- maux une immunodépression importante. On connaissait déjà à cette époque un rétrovirus humain, cause d'une leucémie à cellules $\mathrm{T}$ de l'adulte, le human $T$ cell leukemia virus (HTLV-I) [3]. Les chercheurs américains ont longtemps cru au rôle de ce virus dans le développement de la maladie, d'autant qu'il avait pu être isolé chez quelques malades américains et que 20 à $30 \%$ des sérums de malades atteints de SIDA réagissaient avec un antigène présent à la surface de cellules infectées par ce virus.

A la même époque, le LAV (lymphadenopathy associated virus) était isolé à l'Institut Pasteur à partir d'une culture de lymphocytes ganglionnaires d'un malade souffrant d'adénopathie généralisée [4]. On savait déjà à cette époque que ce syndrome d'adénopathie généralisée associée ou non à des signes généraux non spécifiques tels que fièvre, diarrhée..., était lié au SIDA, pouvant représenter une forme mineure ou précoce de la maladie. Depuis, le même virus a pu être isolé à partir des lymphocytes de malades présentant différents aspects cliniques du SIDA et appartenant aux différents groupes à risques dans lesquels la maladie s'est développée [5]. On sait maintenant qu'il s'agit effectivement de l'agent étiologique de la maladie, bien que de nombreux cofacteurs jouent probablement un rôle dans la physiopathologie et dans l'évolution de celle-ci, après la première infection par le LAV.

\section{Analyse morphologique} du virus

Trois aspects peuvent être observés par l'analyse au microscope électronique de cellules produisant le virus: des particules immatures bourgeonnant à la surface cellulaire avec un croissant dense proche de la membrane plasmique mais qui en est distinctement séparé; des particules immatures libérées de la membrane cellulaire avec un nucléoïde (core) immature en forme d'anneau ou de croissant; enfin des particules matures présentant un petit nucléoïde dense et excentré d'un diamètre moyen de $4 \mathrm{I} \mathrm{nm}$ (figure 1) [5]. Cette morphologie est très différente de celle observée 

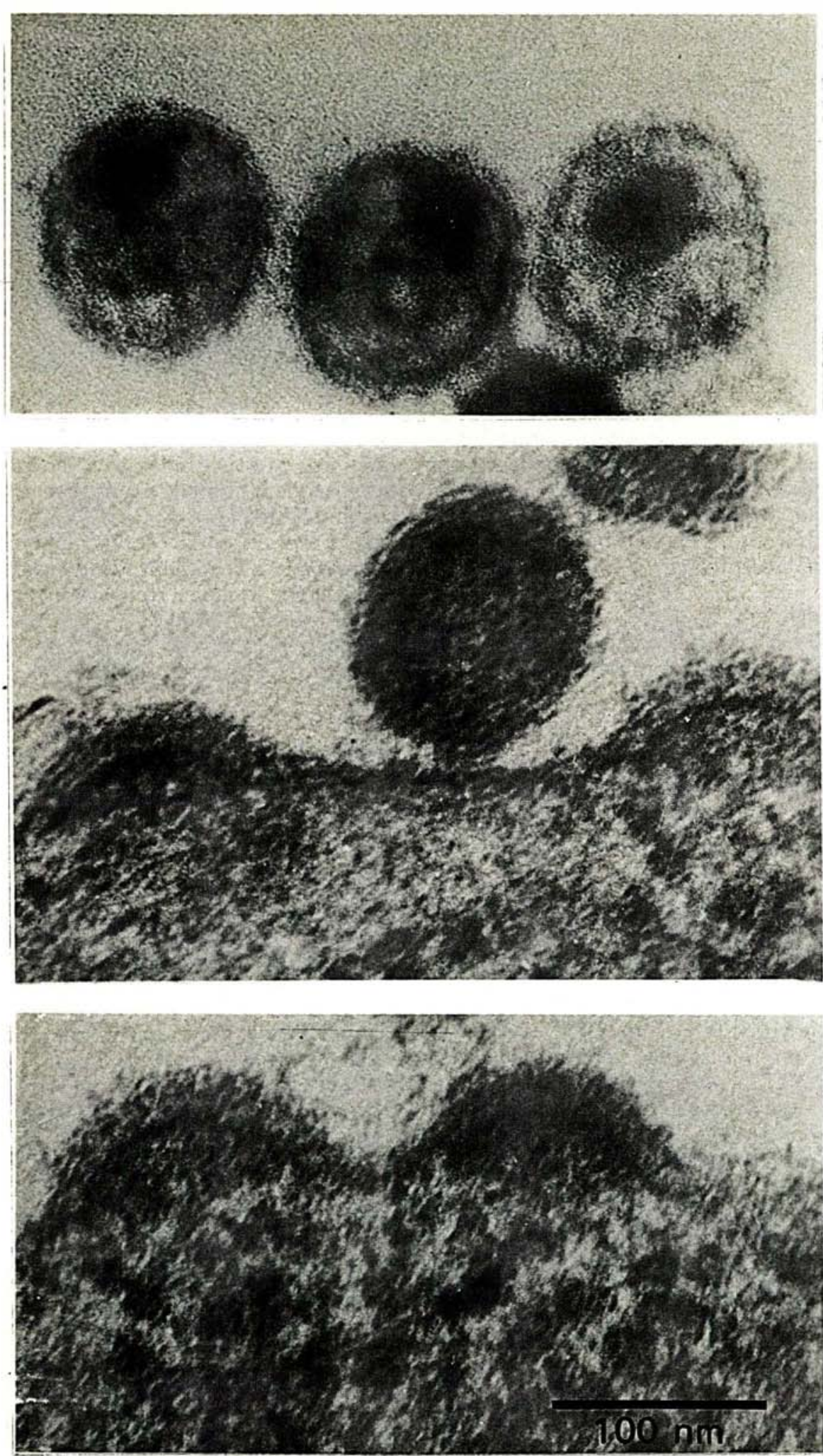

Figure 1 Le LAV, agent étiologique du SIDA : morphologie au microscope électronique.

a. particules immatures bourgeonnant à la surface cellulaire.

b. particules immatures libérées de la membrane cellulaire avec un nucléoïde immature en forme d'anneau ou de croissant.

c. particules matures.

$\mathrm{m} / \mathrm{s}$ mai 85 pour les HTLV-I et -II [6] qui possèdent un nucléoïde central de plus grande taille $(92 \mathrm{~nm})$. L'aspect des particules matures du LAV ressemble plus à celui des particules de type $\mathrm{D}$ des rétrovirus des primates (Mason-Pfizer) ou de celui des virions de l'anémie infectieuse du cheval (EIAV).

\section{Les protéines virales}

Les trois protéines majeures constituant le nucléoïde du LAV (tableau I) ont respectivement un poids moléculaire de 25, i 8 et I3 kD. Elles sont synthétisées à partir du gène gag sous la forme d'un précurseur unique d'un poids moléculaire de $55 \mathrm{kD}$. Comme pour d'autres rétrovirus, ce précurseur est probablement clivé par une protéase d'origine virale qui serait codée par une séquence génomique située au tout début du gène pol. La glycoprotéine (gp) d'enveloppe a un poids moléculaire de I IO kD, c'està-dire presque deux fois supérieur à celui de la glycoprotéine d'enveloppe des HTLV [7]. Cette taille est proche de celle des gp des rétrovirus de mouton ou de chèvre comme le lentivirus Visna. Toutes ces protéines sont immunogènes chez les malades ou les "porteurs sains". Le titre des anticorps du sérum peut diminuer au cours de l'évolution de la maladie et devenir indétectable à sa phase terminale. Les anticorps anti-gp i ro semblent les plus constants [7]. Des protéines de plus petit poids moléculaire $(34,4 \mathrm{I}$ et $70 \mathrm{kD}$ ) peuvent être précipitées par des sérums anti-gp I Io. Elles sont sans doute les produits de clivage de la gp i Io.

\section{- Structure du génome -}

Le clonage du LAV [8] ainsi que l'analyse de la séquence génomique [9] ont été récemment réalisés. Le génome est long de 9,2 kilobases et contient les gènes classiques des rétrovirus: gag, pol, env. L'analyse de la séquence a également permis de mettre en évidence deux nouvelles "phases ouvertes de lecture", appelées respectivement $Q$ et $F$. L'une se trouve entre les gènes pol et env, l'autre entre env et les 


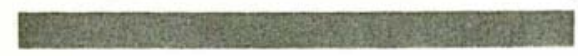

\begin{tabular}{|c|c|c|c|}
\hline \multicolumn{4}{|c|}{$\begin{array}{c}\text { Tableau I } \\
\text { PROTÉINES STRUCTURALES DU LAV }\end{array}$} \\
\hline désignation & $\begin{array}{c}\text { poids } \\
\text { moléculaire }\end{array}$ & localisation & antigénicité \\
\hline $\begin{array}{l}\text { p } 18 \\
\text { p } 25 \\
\text { p } 13 \\
\text { gp } 110\end{array}$ & $\begin{array}{r}18000 \\
25000 \\
13000 \\
110000\end{array}$ & $\begin{array}{l}\text { nucléoïde } \\
\text { nucléoïde } \\
\text { nucléoïde } \\
\text { enveloppe }\end{array}$ & $\begin{array}{c}++ \\
++ \\
+ \\
+++\end{array}$ \\
\hline
\end{tabular}

* poids moléculaire apparent en électrophorèse sur gel de polyacrylamide-SDS.

\section{REFERENCES}

10. Gallo RC, Salahuddin SZ, Popovic M, et al. Frequent detection and isolation of cytopathic retroviruses (HTLV-III) from patients with AIDS and at risk for AIDS. Science 1984; 224: 500-3.

I I. Sarngadharan MG, Popovic M, Bruch L, Schüpbach J, Gallo RC. Antibodies reactive with human T-lymphotropic retroviruses (HTLV-III) in the serum of patients with AIDS. Science 1984 ; 224: 506-8.

12. Kitchen LW, Barin F, Sullivan JL, et al. Aetiology of AIDS-antibodies to human T-cell leukcmia virus (Type-III) in haemophiliacs. Nature 1984; 31 2: 367-9.

13. Levy JA, Hoffmann AD, Kramer SM, Shimabukuto JM, Oshiro LS. Isolation of lymphocytopathic retroviruses from San Francisco patients with AIDS. Science 1984; 225: 840-2.

14. Montagnier L, Dauguet C, Axler C, et al. A new type of retrovirus isolated from patients presenting with lymphadenopathy and acquired immune deficiency syndrome: structural and antigenic relatedness with equine inf ectious anemia virus. Ann Virol (Inst. Pasteur) 1984; $135 \mathrm{E}$ : II 9-34.

15. Klatzmann D, Barré-Sinoussi F, Nugeyre MT, et al. Selective tropism of lymphadenopathy associated virus (LAV) for helper-inducer T-lymphocytes. Science 1984; 225: 59-63.

I6. Klatzmann D, Champagne E, Chamaret $S$, et al. T-lymphocyte $\mathrm{T}_{4}$ molecule behaves as the receptor for human retrovirus LAV. Nature 1984 ; 312: $767-8$.

17. Brun-Vézinet F, Barré-Sinoussi F, Saimot AG, et al. Détection of IgG antibodies to lymphadenopathy associated virus (LAV) by ELISA, in patients with acquired immunodeficiency syndrome or lymphadenopathy syndrome. Lancet 1984; $i$ : $1253-6$

18. Brun-Vézinct F, Rouzioux C, Montagnicr L et al. Prevalence of antibodies to lymphadenopathy associated retrovirus in African patients with AIDS. Science 1984; 226: 453-6.

19. Rouzioux C, Brun-Vézinet F, Courroucé $A M$, et al. IgG antibodies to LAV in differently treated French and Belgian hemophiliacs. Ann

séquences répétées terminales (LTR). Ces régions coderaient pour deux protéines actuellement non identifiées, qui pourraient jouer un rôle dans l'expression et la pathogénicité du virus.

\section{LAV et}

\section{autres rétrovirus}

Aucune réaction croisée n'a pu être détectée entre les anticorps dirigés contre les protéines du nucléoïde du LAV ou des HTLV-I et -II. Les autres protéines sont très différentes, aussi bien en taille qu'en séquences d'acides aminés. Les seules homologies significatives entre les séquences des HTLV-I et -II et celle du LAV sont trouvées dans la région qui code pour la polymérase. Mais cette région est la plus conservée parmi tous les rétrovirus et l'homologie est plus grande avec le virus du sarcome de Rous (38\%) (un virus $\mathrm{du}$ poulet) qu'avec l'HTLV-I (28\%).

Les échanges des différents isolats de HTLV-III, ARV et LAV, ainsi que les séquences, ont clairement établi que ces virus étaient extrêmement proches les uns des autres, voire identiques entre eux pour HTLV-III et LAV [10-I3]. On admet donc qu'il n'y a qu'un seul groupe de rétrovirus à l'origine du SIDA.

Il existe des analogies entre le LAV et certains rétrovirus animaux. Ainsi, on a observé une réactivité croisée entre p 25 du LAV et p 26 de l'EIAV; les sérums des chevaux infectés par l'EIAV peuvent précipiter la p 25 du LAV [14]. Aucune réaction de ce type n'a jusqu'ici été détectée pour les autres protéines du LAV. Les séquences régulatrices de l'ADN proviral du LAV ont des homologies structurales avec celles du virus de la tumeur mammaire de la souris (MMTV).

Les propriétés que nous allons maintenant décrire pour le LAV ont naturellement été retrouvées pour l'HTLV-III et l'ARV.

\section{- Propriétés biologiques}

Les seules cellules humaines normales capables d'être infectées par le LAV puis de permettre sa réplication sont les lymphocytes $\mathrm{T}_{4}{ }^{+}$[ ${ }^{2}$ ] La production virale nécessite l'activation et la multiplication de ces lymphocytes dans la culture. Celles-ci peuvent être obtenues par stimulation par des mitogènes, des allo-antigènes, mais aussi par des antigènes extraits de parois bactériennes ou de virus. La production virale est également favorisée par la présence, dans le milieu de culture, d'anticorps capables de neutraliser l'interféron alpha produit par les lymphocytes infectés. La production virale dans ces cultures est toujours transitoire et n'a jamais abouti à l'apparition de cellules immortalisées comme cela est le cas avec les HTLV-I et -II. Au contraire, cette production virale dans la culture est suivie par une diminution de la prolifération cellulaire puis par un effet cytopathogène important qui se manifeste par l'apparition de cellules géantes résultant de la fusion de plusieurs lymphocytes [15]. En microscopie électronique, on peut voir à la surface de ces cellules des images typiques de bourgeonnement du virus ainsi que des particules virales " matures". 
Enfin, on a observé qu'à la surface des cellules infectées, la molécule $\mathrm{T}_{4}$ devenait progressivement indétectable par les anticorps monoclonaux spécifiqưes de cette molécule. Ce phénomène, qui est contemporain de la production virale, a conduit à supposer qu'il existait une interaction directe entre la glycoprotéine d'enveloppe des particules virales et la molécule $\mathrm{T}_{4}$. En effet, nous avons pu montrer que cette molécule jouait le rôle de récepteur pour le virus à la surface des lymphocytes $\mathrm{T}_{4}$. L'incubation de ces lymphocytes avec des anticorps monoclonaux anti- $\mathrm{T}_{4}$ masque cette molécule à la surface des lymphocytes et empêche la pénétration du virus, protégeant ainsi les cellules d'une infection par le LAV [16].

\section{Les anticorps} anti-LAV

Quatre méthodologies différentes peuvent être utilisées pour détecter les anticorps anti-LAV dans le sérum des malades. L'immunofluorescence sur des lignées cellulaires infectées par le LAV, le test ELISA réalisé à partir de virus purifiés, le Western blot et la RIPA. Seules les deux dernières techniques permettent de préciser la présence d'anticorps cóntre chacune des protéines du nucléoïde et la protéine d'enveloppe du virus. Les résultats obtenus avec ces différentes techniques sont les suivants (tableau II et $[17$, I 8, 19]) :

Chez les patients atteints du SIDA, les études séquentielles ont pu montrer une évolution du titre des anticorps lors de l'évolution de la maladie. Au stade ultime, il peut $\mathrm{y}$ avoir disparition des anticorps dirigés contre les protéines du nucléoïde du virus mais en général les anticorps dirigés contre la gp i io restent détectables par RIPA.

Les patients présentant des lymphadénopathies généralisées avec ou sans signes généraux d'accompagnement ont habituellement une réponse d'anticorps plus forte que celle des patients ayant un SIDA vrai. Les anticorps anti-p 25 peuvent être détectés chez environ $70 \%$ de ces patients, et environ $90 \%$ sont positifs en ELISA. Certains n'ont des anticorps détectables que contre la gp i 10.

Parmi les personnes saines appartenant aux différents groupes à risques, aux États-Unis (San Francisco), le CDC (Center for Disease Control) a pu étudier la sérologie anti-LAV d'un groupe

Tableau II

ISOLEMENT DU LAV OU D'ANTICORPS ANTI-LAV CHEZ LES MALADES ATTEINTS DE SIDA, PARA-SIDA OU APPARTENANT AUX POPULATIONS A RISQUES

\begin{tabular}{|l|c|c|c|}
\hline & & $\begin{array}{c}\text { isolat } \\
\text { LAV }\end{array}$ & $\begin{array}{c}\text { séropositivité } \\
(\%)\end{array}$ \\
\hline \multirow{2}{*}{ homosexuels } & $\begin{array}{c}\text { sains } \\
\text { polyadénopathies } \\
\text { SIDA }\end{array}$ & $\begin{array}{c}\text { NT } \\
+ \\
+\end{array}$ & $\begin{array}{c}25-60(a) \\
90-95(b) \\
75-90(b)\end{array}$ \\
\hline \multirow{2}{*}{ hémophiles } & Sains & + & $5-60(c)$ \\
& SIDA & + & $90-95$ \\
\hline \multirow{2}{*}{ africains } & Sains & NT & $10-12(d)$ \\
& SIDA & + & $90-95(d)$ \\
\hline \multirow{2}{*}{ haïtiens } & Sains & + & NT \\
\hline témoins & SIDA & + & $90-95(b)$ \\
\hline
\end{tabular}

(a) Variation selon le pays d'origine et la ville. (b) Variation selon le test utilisé - la détection de la gp 110 augmente la fréquence de séropositivité. (c) Variations selon les centres, l'intensité du traitement et l'origine des produits sanguins [16]. (d) réf. $|7|$. NT: Non testé.

$\mathrm{m} / \mathrm{s}$ mai 85 d'homosexuels suivis depuis 1978 . A cette époque, I \% avait des anticorps anti-p 25. En I $98 \mathrm{I}, 24 \%$ avaient développé des anticorps et en $1984,60 \%$. Une telle progression de la fréquence des personnes porteuses d'anticorps anti-LAV est observée également dans d'autres groupes à risque comme les utilisateurs de drogues par voie intraveineuse ou les hémophiles. En France, une étude prospective réalisée en I 984 , dans un groupe d'homosexuels en bonne santé mais consultant un dispensaire pour maladies sexuellement transmissibles, a montré une fréquence d'anticorps anti-LAV d'environ $20 \%$. Ces résultats ne sont pas généralisables à la population homosexuelle française, car ils ne concernent que les homosexuels parisiens et le mode de recrutement introduit certainement un biais.

Une étude réalisée dans plusieurs centres traitant des hémophiles français à montré que le pourcentage des malades porteurs d'anticorps anti-LAV dépendait de la provenance et de la quantité des préparations de facteur VIII et facteur IX utilisés. Dans certains centres, $60 \%$ d'hémophiles étaient porteurs d'anticorps anti-LAV tandis que dans d'autres, utilisant des protocoles thérapeutiques plus légers, seulement I $\%$ étaient positifs [I9].

En ce qui concerne la population hétérosexuelle en général, les études en cours montrent qu'il existe moins de I \% de personnes porteuses d'anticorps anti-LAV parmi les donneurs de sang volontaires.

Si la présence d'anticorps anti-LAV traduit sans nul doute le contact de la personne avec le virus, il ne renseigne pas sur l'évolution future de la maladie, ni sur la contagiosité éventuelle de cette personne. La mise au point de tests détectant les anticorps anti-LAV de type $\operatorname{IgM}$, ainsi que des tests permettant de détecter l'antigène viral lui-même dans le sérum ou dans les cellules infectées, devrait résoudre en partie ce problème.

Le SIDA se présente donc bien comme une maladie immunologique due à un rétrovirus. Sa physiopathologie commence par la propriété surprenante de la glyco- 
protéine d'enveloppe du LAV de se lier à la molécule $T_{4}$, qui lui sert ainsi de récepteur et permet sa pénétration dans une catégorie particulière de cellules ayant un rôle fondamental dans le développement de la réponse immunitaire.

\section{Un modèle}

\section{physiopathologique}

Ensuite, l'infection virale contribue à lyser directement les cellules infectées et, probablement par des mécanismes indirects, va éliminer un plus grand nombre de cellules $T_{4}$. Au cours de l'évolution de cette infection, le nombre des lymphocytes $\mathrm{T}_{4}$ va décroître progressivement et leur fonction s'altérer. Ainsi lorsque ceux-ci ne seront plus en nombre suffisant, la moindre rencontre avec un agent infectieux contrôlé par des réponses immunitaires impliquant ces lymphocytes, donnera lieu à une maladie grave. Plusieurs facteurs pourraient être impliqués dans la survenue de la maladie après l'infection, et dans son évolution. Le terrain sur lequel survient l'infection, la susceptibilité individuelle à cet agent infectieux, la voie, la quantité et la répétition des infections, ont probablement un rôle fondamental. Pour ce qui est du terrain, il faut noter que la plupart des populations à risque parmi lesquelles la maladie s'est développée présentent des signes biologiques de déficit immunitaire avant même toute infection par le LAV.

Ces populations sont également soumises à des infections extrêmement fréquentes par des virus communs, des bactéries, des parasites ou des champignons. On peut alors supposer que l'infection primaire par le LAV pourrait être inapparente et ne concernerait qu'une faible fraction des lymphocytes. La répétition de ces infections, la stimulation des lymphocytes infectés par d'autres infections microbiennes plus classiques, ainsi que d'éventuelles stimulations allogéniques de ces lymphocytes par des lymphocytes contenus dans le sperme, pourraient alors contribuer à la diffusion de l'infection dans l'organisme. Enfin la susceptibilité individuelle qui représente la capacité propre d'un individu à répondre à une infection donnée, peut probablement influer sur le cours de la maladie par l'intensité des réponses immunitaires humorales ou cellulaires développées contre le virus.

Bien que des progrès importants aient été obtenus dans la connaissance de l'agent étiologique du SIDA, le LAV, de nombreuses questions restent posées. Concernant le virus lui-même, on sait déjà qu'il est très éloigné de la famille des HTLV et qu'il est plus probablement le prototype d'un nouveau groupe de rétrovirus humains. Cependant, si certaines analogies de sa structure, telle la taille de sa glycoprotéine, le rapprochent de la famille des lentivirus, sa place précise dans la phylogénie des différents rétrovirus humains et animaux n'est pas encore déterminée avec précision. L'analyse de la séquence de ce virus a également démontré l'existence de deux " cadres ouverts de lecture " qui pourraient coder pour des protéines importantes dans la physiologie de la réplication virale et des effets pathogènes du virus, mais qui sont encore inconnus.

La réponse immunitaire contre ce virus est également mal connue. $\mathrm{Si}$ l'on connaît assez précisément les différents types d'anticorps produits par les malades, on n'en connaît pas encore la signification exacte. En particulier, le rôle neutralisant des anticorps dirigés contre la glycoprotéine d'enveloppe gp i I o est encore discuté. L'existence d'une réponse immunitaire de type cellulaire dirigée contre le virus et les cellules infectées n'a pas encore été étudiée, en raison de la difficulté à établir des lignées autologues chroniquement infectées, permettant d'étudier, par exemple, les mécanismes de cytotoxicité cellulaire. La part de l'auto-immunité dans le développement de la maladie est également encore inconnue. Quant au traitement de la maladie ou la fabrication d'un vaccin, ils souffrent de l'absence d'un modèle animal.

La collaboration active entre épidémiologistes, cliniciens, immunologistes et virologistes permettra sans doute de répondre à ces questions comme elle a déjà permis les progrès rapides qui ont abouti à l'isolement et à la caractérisation de son agent étiologique

\section{Summary}

AIDS is a newly described syndrome whose epidemic character appeared in the carly eighties in North America, and then in Western Europe. It occurs also in African and Haitian populations. It is characterized by a profound depression of cellular immunity caused by an absolute and functional deficit of $\mathrm{T}_{4} \mathrm{lym}$ phocytes. These lymphocytes are the preferential target of a new type of human retroviruses whose prototype described in I985 is the lymphadenopathy associated virus (LAV).

Nucleotide sequence data have indicated that this virus does not belong to the HTLV-I and-II family. The etiological role of this virus in AIDS and related syndromes is demonstrated by: I) its frequent isolation from patients with the disease, or belonging to groups with a high risk of acquiring the disease; 2) its biological properties: tropism for $\mathrm{T}_{4}$-lymphocytes with induction of a cytopathogenic effect in vitro; 3) transmission of the virus by blood and blood products; 4) sero-epidemiological data showing a high prevalence of anti-LAV antibodies in patients with AIDS and highrisk populations. The role of cofactors in the pathophysiology of AIDS is likely.

\section{TIRES A PART}

L. Montagnier : Institut Pasteur. 25-28, rue du Docteur-Roux. 75724 Paris Cedex 15. 